\title{
Entanglement in neutrino oscillations
}

\author{
Massimo Blasone ${ }^{1,2}$, Fabio Dell'Anno ${ }^{1,2,3}$, Silvio De Siena ${ }^{1,2,3}$ and Fabrizio Illuminati ${ }^{1,2,3,4}$ \\ 1 Dipartimento di Matematica e Informatica, Università degli Studi di Salerno, Via Ponte don Melillo, I-84084 Fis- \\ ciano (SA), Italy \\ ${ }^{2}$ INFN Sezione di Napoli, Gruppo collegato di Salerno, Baronissi (SA), Italy \\ CNR-INFM Coherentia, Napoli, Italy \\ 4 ISI Foundation for Scientific Interchange, Viale Settimio Severo 65, I-10133 Torino, Italy
}

PACS 03.65.Ud - Entanglement and quantum nonlocality

PACS 03.67.Mn - Entanglement production, characterization, and manipulation

PACS $14.60 . \mathrm{Pq}$ - Neutrino mass and mixing

\begin{abstract}
Flavor oscillations in elementary particle physics are related to multi-mode entanglement of single-particle states. We show that mode entanglement can be expressed in terms of flavor transition probabilities, and therefore that single-particle entangled states acquire a precise operational characterization in the context of particle mixing. We treat in detail the physically relevant cases of two- and three-flavor neutrino oscillations, including the effective measure of $C P$ violation. We discuss experimental schemes for the transfer of the quantum information encoded in single-neutrino states to spatially delocalized two-flavor charged lepton states, thus showing, at least in principle, that single-particle entangled states of neutrino mixing are legitimate physical resources for quantum information tasks.
\end{abstract}

Various branches of condensed matter, atomic physics, and quantum optics have evolved in recent years towards the investigation and development of schemes for quantum information and computation science [1]. To this aim, entanglement is a key ingredient and a crucial physical resource. Different forms of entanglement have been proven to be equivalent to observable quantifiers of performance success in quantum information protocols either with discrete [2] or continuous variables [3]. In the present work, we investigate the operational meaning of entanglement in the context of elementary particles physics. We will show that single-particle (mode) entanglement associated to particle mixing can be expressed in terms of transition probabilities in flavor oscillations, and can be exploited for quantum informational tasks.

The concept of single-particle entanglement has been introduced and elucidated in a recent series of important theoretical papers [4-6]. Its use has been discussed in various contexts of quantum information, including teleportation, quantum cryptography, and violation of Bell inequalities $[7,8]$, and later experimentally demonstrated with single-photon systems [9-11]. Existing schemes to probe non-locality in single-photon states have been subsequently generalized to include massive particles [12]. In the present work we extend the discussion to the arena of elementary particles and provide a general operational characterization of single-particle entanglement in this context by showing its connection with the transition probabilities in any quantum system oscillating between different modes. We then show how this form of entanglement is in principle a real physical resource for the realization of quantum information protocols by discussing explicit experimental schemes for transferring it to spatially separated modes of stable leptonic particles. These results allow to place mode entanglement in neutrino oscillations on equal footing with mode entanglement in single-particle atomic and optical systems. Furthermore, we show how the single-particle entanglement quantifies $C P$ violation in neutrino mixing.

Flavor mixing of neutrinos for three generations is described by the $3 \times 3$ Pontecorvo-Maki-Nakagawa-Sakata (PMNS) unitary mixing matrix $\mathbf{U}(\tilde{\theta}, \delta)[13]$, where $(\tilde{\theta}, \delta) \equiv\left(\theta_{12}, \theta_{13}, \theta_{23} ; \delta\right)$ and $c_{i j} \equiv \cos \theta_{i j}, s_{i j} \equiv \sin \theta_{i j}$. The parameters $\theta_{i j}$ are the mixing angles, and $\delta$ is the phase responsible for $C P$ violation. Here, without loss of generality we consider only Dirac neutrinos. In the instance of Majorana neutrinos, two additional CP-violating phases are present, which, however, as it is well known, do not affect the physics of neutrino oscillations. The three- 


$$
\mathbf{U}(\tilde{\theta}, \delta)=\left(\begin{array}{ccc}
c_{12} c_{13} & s_{12} c_{13} & s_{13} e^{-i \delta} \\
-s_{12} c_{23}-c_{12} s_{23} s_{13} e^{i \delta} & c_{12} c_{23}-s_{12} s_{23} s_{13} e^{i \delta} & s_{23} c_{13} \\
s_{12} s_{23}-c_{12} c_{23} s_{13} e^{i \delta} & -c_{12} s_{23}-s_{12} c_{23} s_{13} e^{i \delta} & c_{23} c_{13}
\end{array}\right)
$$

flavor neutrino states are defined as

$$
\left|\underline{\nu}^{(f)}\right\rangle=\mathbf{U}(\tilde{\theta}, \delta)\left|\underline{\nu}^{(m)}\right\rangle
$$

where $\left|\underline{\nu}^{(f)}\right\rangle=\left(\left|\nu_{e}\right\rangle,\left|\nu_{\mu}\right\rangle,\left|\nu_{\tau}\right\rangle\right)^{T}$ are the states with definite flavor and $\left|\underline{\nu}^{(m)}\right\rangle=\left(\left|\nu_{1}\right\rangle,\left|\nu_{2}\right\rangle,\left|\nu_{3}\right\rangle\right)^{T}$ those with definite mass. Let us recall that both $\left|\nu_{\alpha}\right\rangle(\alpha=e, \mu, \tau)$ and $\left|\nu_{j}\right\rangle(j=1,2,3)$ are orthonormal, i.e. $\left\langle\nu_{\alpha} \mid \nu_{\beta}\right\rangle=\delta_{\alpha, \beta}$ and $\left\langle\nu_{j} \mid \nu_{k}\right\rangle=\delta_{j, k}$.

Neutrino oscillations are due to neutrino mixing and neutrino mass differences. The neutrino states $\left|\nu_{j}\right\rangle$ have definite masses $m_{j}$ and definite energies $E_{j}$. Their propagation can be described by plane wave solutions of the form $\left|\nu_{j}(t)\right\rangle=e^{-i E_{j} t}\left|\nu_{j}\right\rangle$. The time evolution of the flavor neutrino states is given by:

$$
\begin{aligned}
\left|\underline{\nu}^{(f)}(t)\right\rangle & =\widetilde{\mathbf{U}}(t)\left|\underline{\nu}^{(f)}\right\rangle, \\
\widetilde{\mathbf{U}}(t) & \equiv \mathbf{U}(\tilde{\theta}, \delta) \mathbf{U}_{0}(t) \mathbf{U}(\tilde{\theta}, \delta)^{-1},
\end{aligned}
$$

where $\left|\underline{\nu}^{(f)}\right\rangle$ are the flavor states at $t=0, \mathbf{U}_{0}(t)=$ $\operatorname{diag}\left(e^{-i E_{1} t}, e^{-i E_{2} t}, e^{-i E_{3} t}\right)$, and $\widetilde{\mathbf{U}}(t=0)=\mathbb{I}$. At time $t$ the transition probability for $\nu_{\alpha} \rightarrow \nu_{\beta}$ is

$$
P_{\nu_{\alpha} \rightarrow \nu_{\beta}}(t)=\left|\left\langle\nu_{\beta} \mid \nu_{\alpha}(t)\right\rangle\right|^{2}=\left|\widetilde{\mathbf{U}}_{\alpha \beta}(t)\right|^{2},
$$

where $\alpha, \beta=e, \mu, \tau$. The transition probability $P_{\nu_{\alpha} \rightarrow \nu_{\beta}}(t)$ is a function of the energy differences $\Delta E_{j k}=E_{j}-E_{k}$ $(j, k=1,2,3)$ and of the mixing angles. Since the current neutrino experiments deal with ultra-relativistic neutrinos, the standard adopted approximation is $\Delta E_{j k} \simeq$ $\frac{\Delta m_{j k}^{2}}{2 E}$, where $\Delta m_{j k}^{2}=m_{j}^{2}-m_{k}^{2}$ and $E=|\vec{p}|$ is the energy of a massless neutrino (all massive neutrinos are assumed to have the same momentum $\vec{p}$ ).

Flavor neutrinos are identified via charged current weak interaction processes, together with their associated charged leptons. In the Standard Model (SM), where neutrinos are taken to be massless, flavor is strictly conserved in such processes. On the other hand, neutrino mixing, consisting in a mismatch between flavor and mass, is at the basis of neutrino oscillations and $\mathrm{CP}$ violation. The introduction of neutrino masses as a correction to the SM is a necessary condition to explain such effects.

When neutrino mixing is considered, loop corrections produce violations of lepton flavor in the charged current vertices: however, these effects are extremely small and essentially vanish in the relativistic limit [14]. Consequently, neutrino states entering weak interaction processes, like the ones where flavor neutrinos are created or detected, must be eigenstates of flavor neutrino charges. The corresponding operators can be rigorously defined together with their eigenstates in the context of Quantum Field Theory
(QFT) [15]. In the relativistic limit, the exact QFT flavor states reduce to the usual Pontecorvo flavor states, which define the flavor modes as legitimate and physically welldefined individual entities. Mode entanglement can thus be defined and studied in analogy with the static case [16].

Equipped with the above setting, one can establish the following correspondence with three-qubit states: $\left|\nu_{e}\right\rangle \equiv|1\rangle_{\nu_{e}}|0\rangle_{\nu_{\mu}}|0\rangle_{\nu_{\tau}},\left|\nu_{\mu}\right\rangle \equiv|0\rangle_{\nu_{e}}|1\rangle_{\nu_{\mu}}|0\rangle_{\nu_{\tau}},\left|\nu_{\tau}\right\rangle \equiv$ $|0\rangle_{\nu_{e}}|0\rangle_{\nu_{\mu}}|1\rangle_{\nu_{\tau}}$. States $|0\rangle_{\nu_{\alpha}}$ and $|1\rangle_{\nu_{\alpha}}$ correspond, respectively, to the absence and the presence of a neutrino in mode $\alpha$. Entanglement is thus established among flavor modes, in a single-particle setting. Eq. (3) can then be recast as

$$
\begin{aligned}
\left|\nu_{\alpha}(t)\right\rangle= & \widetilde{\mathbf{U}}_{\alpha e}(t)|1\rangle_{\nu_{e}}|0\rangle_{\nu_{\mu}}|0\rangle_{\nu_{\tau}}+\widetilde{\mathbf{U}}_{\alpha \mu}(t)|0\rangle_{\nu_{e}}|1\rangle_{\nu_{\mu}}|0\rangle_{\nu_{\tau}} \\
& +\widetilde{\mathbf{U}}_{\alpha \tau}(t)|0\rangle_{\nu_{e}}|0\rangle_{\nu_{\mu}}|1\rangle_{\nu_{\tau}},
\end{aligned}
$$

with the normalization condition $\sum_{\beta}\left|\widetilde{\mathbf{U}}_{\alpha \beta}(t)\right|^{2}=1$ $(\alpha, \beta=e, \mu, \tau)$. The time-evolved states $\left|\underline{\nu}^{(f)}(t)\right\rangle$ are entangled superpositions of the three flavor eigenstates with time-dependent coefficients. It is important to remark that, although dealing with similar physical systems, both the framework and the aim of the present paper differ substantially from those of Ref. [16]. In the latter, by exploiting the wave packet approach, the multipartite entanglement, associated with the multiqubit space of mass modes, has been analyzed in connection with the "decoherence" effects induced by free evolution. In the present work, by exploiting the plane-wave approximation, the entanglement is quantified with respect to the multiqubit space associated with flavor modes, and is related to the quantum information encoded in the neutrino flavor states, which is in principle experimentally accessible, as we will show by devising an explicit scheme for the information transfer.

States of the form Eq. (5) belong to the class of $W$ states. These, together with the $G H Z$ states, define the two possible sets of states with tripartite entanglement that are inequivalent under local operations and classical communication [17]. In some instances, only two neutrinos are significantly involved in the mixing. For example, only the transition $\nu_{\mu} \leftrightarrow \nu_{\tau}$ is relevant for atmospheric neutrinos, while only the transitions of the type $\nu_{e} \leftrightarrow \nu_{\alpha}$ are relevant for solar neutrinos. For two-flavor mixing the mixing matrix $\mathbf{U}(\tilde{\theta}, \delta)$ reduces to the $2 \times 2$ rotation matrix $\mathbf{U}(\theta)$,

$$
\mathbf{U}(\theta)=\left(\begin{array}{cc}
\cos \theta & \sin \theta \\
-\sin \theta & \cos \theta
\end{array}\right),
$$

the evolution operator reads $\mathbf{U}_{0}(t)=\operatorname{diag}\left(e^{-i E_{1} t}, e^{-i E_{2} t}\right)$, and the time-evolved flavor states yield the Bell-like superposition $(\alpha=e, \mu)$ :

$$
\left|\nu_{\alpha}(t)\right\rangle=\widetilde{\mathbf{U}}_{\alpha e}(t)|1\rangle_{\nu_{e}}|0\rangle_{\nu_{\mu}}+\widetilde{\mathbf{U}}_{\alpha \mu}(t)|0\rangle_{\nu_{e}}|1\rangle_{\nu_{\mu}} .
$$


Bipartite entanglement of pure states is unambiguously quantified by the von Neumann entropy or by any other monotonic function of the former [18]. Among entanglement monotones, the linear entropy has a special physical significance because it is directly linked to the purity of the reduced states, and enters in the fundamental monogamy inequalities for distributed entanglement in the multipartite setting [18]. As one moves from the two- to the three-flavor scenario, multipartite entanglement measures are readily available in terms of functions of bipartite measures [19-21]. Representative of this type of measures is the global entanglement. It is defined as the sum of all the two-qubit entanglements between a single subsystem and each of the remaining ones [19], and can be expressed as the average subsystem linear entropy [20]. Global entanglement can then be generalized by constructing the set of mean linear entropies associated to all possible bi-partitions of the entire system [21]. An alternative characterization of multipartite entanglement is given in Refs. [22, 23].

Let $\rho=|\psi\rangle\langle\psi|$ be the density operator corresponding to a pure state $|\psi\rangle$, describing the system $S$ partitioned into $N$ parties. Consider the bipartition of the $N$-partite system $S=\left\{S_{1}, S_{2}, \ldots, S_{N}\right\}$ in two subsystems $S_{A_{n}}=$ $\left\{S_{i_{1}}, S_{i_{2}}, \ldots, S_{i_{n}}\right\}$, with $1 \leq i_{1}<i_{2}<\ldots<i_{n} \leq N$ $(1 \leq n<N)$, and $S_{B_{N-n}}=\left\{S_{j_{1}}, S_{j_{2}}, \ldots, S_{j_{N-n}}\right\}$, with $1 \leq j_{1}<j_{2}<\ldots<j_{N-n} \leq N$, and $i_{q} \neq j_{p}$. Let

$$
\rho_{A_{n}} \equiv \rho_{i_{1}, i_{2}, \ldots, i_{n}}=\operatorname{Tr}_{B_{N-n}}[\rho]=\operatorname{Tr}_{j_{1}, j_{2}, \ldots, j_{N-n}}[\rho]
$$

denote the reduced density matrix of subsystem $S_{A_{n}}$ after tracing over subsystem $S_{B_{N-n}}$. The linear entropy associated to such a bipartition is defined as

$$
S_{L}^{\left(A_{n} ; B_{N-n}\right)}(\rho)=\frac{d}{d-1}\left(1-\operatorname{Tr}_{A_{n}}\left[\rho_{A_{n}}^{2}\right]\right),
$$

where the $d$ is the Hilbert-space dimension given by $d=$ $\min \left\{\operatorname{dim} S_{A_{n}}, \operatorname{dim} S_{B_{N-n}}\right\}=\min \left\{2^{n}, 2^{N-n}\right\}$. Finally, we introduce the average linear entropy

$$
\left\langle S_{L}^{(n: N-n)}(\rho)\right\rangle=\left(\begin{array}{c}
N \\
n
\end{array}\right)^{-1} \sum_{A_{n}} S_{L}^{\left(A_{n} ; B_{N-n}\right)}(\rho)
$$

where the sum is intended over all the possible bipartitions of the system in two subsystems, respectively with $n$ and $N-n$ elements $(1 \leq n<N)$ [21].

We can now compute the linear entropies (9) and (10) for the two-qubit Bell state $\left|\nu_{\alpha}(t)\right\rangle$, i.e. Eq. (7), with density matrix $\rho^{(\alpha)}=\left|\nu_{\alpha}(t)\right\rangle\left\langle\nu_{\alpha}(t)\right|$. The linear entropy associated to the reduced state after tracing over one mode (flavor) can be computed straightforwardly:

$$
\begin{aligned}
S_{L \alpha}^{(\mu ; e)}=S_{L \alpha}^{(e ; \mu)} & =4\left|\widetilde{\mathbf{U}}_{\alpha e}(t)\right|^{2}\left|\widetilde{\mathbf{U}}_{\alpha \mu}(t)\right|^{2} \\
& =4\left|\widetilde{\mathbf{U}}_{\alpha e}(t)\right|^{2}\left(1-\left|\widetilde{\mathbf{U}}_{\alpha e}(t)\right|^{2}\right) \\
& =4\left|\widetilde{\mathbf{U}}_{\alpha \mu}(t)\right|^{2}\left(1-\left|\widetilde{\mathbf{U}}_{\alpha \mu}(t)\right|^{2}\right)
\end{aligned}
$$

In Eq. (11) and in the following, we use the notation $S_{L \alpha}^{(e ; \mu)} \equiv S_{L}^{(e ; \mu)}\left(\rho^{(\alpha)}\right)$, where the subscript $\alpha$ refers to the time-evolved state (channel), and the superscripts $(e ; \mu)$ refer to the considered modes (flavors). Clearly, for the two-flavor state (7), and in general for any two-qubit system, symmetry imposes $S_{L \alpha}^{(e ; \mu)}=S_{L \alpha}^{(\mu ; e)}=\left\langle S_{L \alpha}^{(1: 1)}\right\rangle$. Expression (11) establishes that the linear entropy of the reduced state is equal to the product of the two-flavor transition probabilities. Moreover, for any reduced state $\rho$ of a two-level system one has that $S_{L}=2\left[1-\operatorname{Tr}\left(\rho^{2}\right)\right]=$ $4 \operatorname{Det} \rho=4 \lambda_{1}\left(1-\lambda_{1}\right)$, where $\lambda_{1}$ is one of the two nonnegative eigenvalues of $\rho$, and the relation $\lambda_{1}+\lambda_{2}=1$ has been exploited. Comparing with Eq. (11), one sees that the transition probabilities coincide with the eigenvalues of the reduced state density matrix.

In Fig. 1 we show the behavior of $S_{L e}^{(e ; \mu)}$ as a function of the scaled, dimensionless time $T=\frac{2 E t}{\Delta m_{12}^{2}}$. In the same figure, we also report the behavior of the transition probabilities $P_{\nu_{e} \rightarrow \nu_{e}}$ and $P_{\nu_{e} \rightarrow \nu_{\mu}}$. The plots have a clear physical

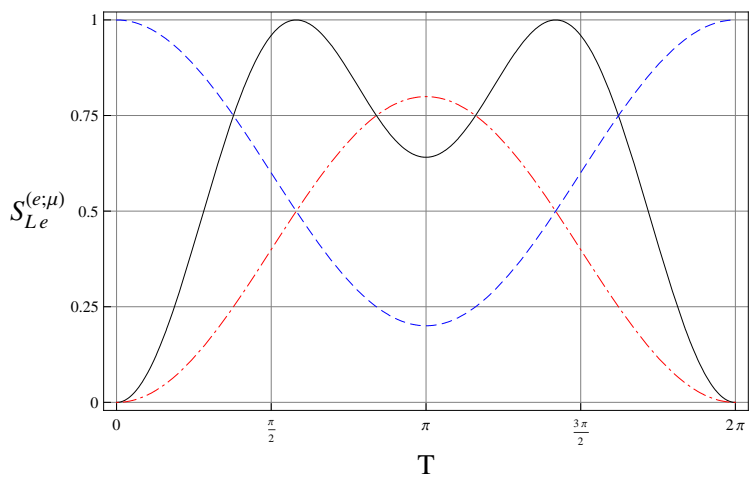

Fig. 1: (Color online) Linear entropy $S_{L e}^{(e ; \mu)}$ (full) as a function of the scaled time $T=\frac{2 E t}{\Delta m_{12}^{2}}$. The mixing angle $\theta$ is fixed at the experimental value $\sin ^{2} \theta=0.314$. The transition probabilities $P_{\nu_{e} \rightarrow \nu_{e}}$ (dashed) and $P_{\nu_{e} \rightarrow \nu_{\mu}}$ (dot-dashed) are reported as well for comparison.

interpretation. At time $T=0$, the entanglement is zero, the global state of the system is factorized, and the two flavors are not mixed. For $T>0$, flavors start to oscillate and the entanglement is maximal at largest mixing: $P_{\nu_{e} \rightarrow \nu_{e}}=P_{\nu_{e} \rightarrow \nu_{\mu}}=0.5$, and minimum at $T=\pi$.

We can now investigate three-flavor oscillations, and the associated three-qubit $W$-like states (5). Similarly to Eq. (11), tracing, e. g., over mode $\tau$, one has

$$
\begin{aligned}
S_{L \alpha}^{(e, \mu ; \tau)} & =4\left|\widetilde{\mathbf{U}}_{\alpha \tau}(t)\right|^{2}\left(\left|\widetilde{\mathbf{U}}_{\alpha e}(t)\right|^{2}+\left|\widetilde{\mathbf{U}}_{\alpha \mu}(t)\right|^{2}\right) \\
& =4\left|\widetilde{\mathbf{U}}_{\alpha \tau}(t)\right|^{2}\left(1-\left|\widetilde{\mathbf{U}}_{\alpha \tau}(t)\right|^{2}\right)
\end{aligned}
$$

The linear entropies for the two remaining bi-partitions are easily obtained by permuting the indexes $e, \mu, \tau$. The average linear entropy for the state (5) is then

$$
\begin{aligned}
\left\langle S_{L \alpha}^{(2: 1)}\right\rangle= & \frac{8}{3}\left(\left|\widetilde{\mathbf{U}}_{\alpha e}(t)\right|^{2}\left|\widetilde{\mathbf{U}}_{\alpha \mu}(t)\right|^{2}+\left|\widetilde{\mathbf{U}}_{\alpha e}(t)\right|^{2}\left|\widetilde{\mathbf{U}}_{\alpha \tau}(t)\right|^{2}\right. \\
& \left.+\left|\widetilde{\mathbf{U}}_{\alpha \mu}(t)\right|^{2}\left|\widetilde{\mathbf{U}}_{\alpha \tau}(t)\right|^{2}\right) .
\end{aligned}
$$

In Fig. 2] we show $S_{L e}^{(\alpha, \beta ; \gamma)}$ and $\left\langle S_{L e}^{(2 ; 1)}\right\rangle$ as functions of the scaled time $T=\frac{2 E t}{\Delta m_{12}^{2}}$. The mixing angles $\theta_{i j}$ and the 
squared mass differences are fixed at the most recent experimental values reported in Ref. [24]. In order to track

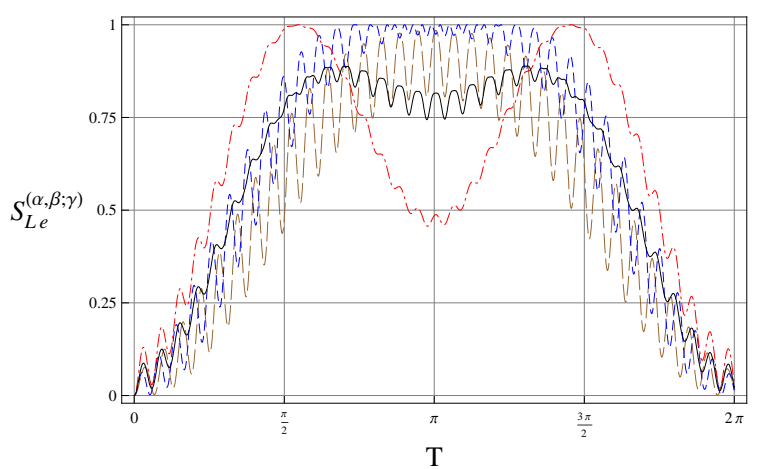

Fig. 2: (Color online) Linear entropies $S_{L e}^{(\alpha, \beta ; \gamma)}$ and $\left\langle S_{L e}^{(2 ; 1)}\right\rangle$ as functions of the scaled time $T$. Curves correspond to the partial linear entropies $S_{L e}^{(e, \mu ; \tau)}$ (long-dashed), $S_{L e}^{(e, \tau ; \mu)}$ (dashed), $S_{L e}^{(\mu, \tau ; e)}$ (dot-dashed), and to the average linear entropy $\left\langle S_{L e}^{(2 ; 1)}\right\rangle$ (full). Parameters $\theta_{i j}$ and $\Delta m_{i j}^{2}$ are fixed at the central experimental values [24].

the behavior of the entanglement, we plot in Fig. 3 the transition probabilities $P_{\nu_{e} \rightarrow \nu_{\alpha}}(\alpha=e, \mu, \tau)$. Comparing

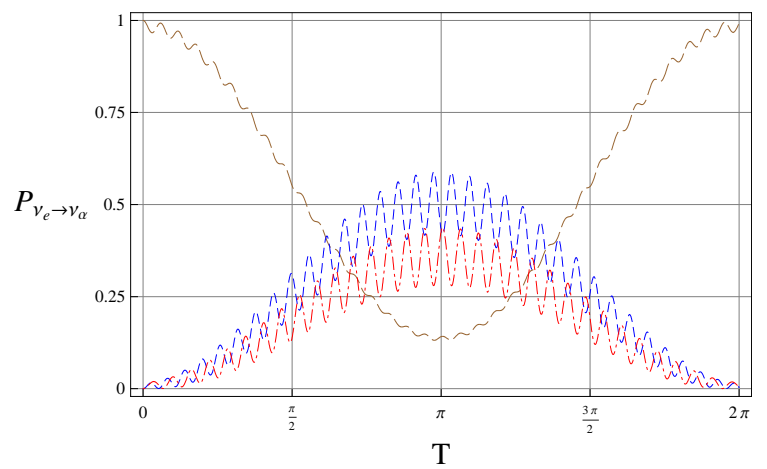

Fig. 3: (Color online) Transition probabilities $P_{\nu_{e} \rightarrow \nu_{\alpha}}$ as functions of the scaled time $T$. Parameters $\theta_{i j}$ and $\Delta m_{i j}^{2}$ are fixed at the central experimental values as in Fig. 2 Curves correspond to $P_{\nu_{e} \rightarrow \nu_{e}}$ (long-dashed), $P_{\nu_{e} \rightarrow \nu_{\mu}}$ (dashed), and $P_{\nu_{e} \rightarrow \nu_{\tau}}$ (dot-dashed).

Fig. 2 and Fig. 3. we observe that, as one may expect, the more mixed are the flavors, the higher is the global multipartite entanglement of the system. Moreover, the partial linear entropies $S_{L e}^{(e, \mu ; \tau)}$ and $S_{L e}^{(e, \tau ; \mu)}$ measuring the reduced bipartite entanglement, exhibit a similar behavior due to the strong correlation between the components $\nu_{\mu}$ and $\nu_{\tau}$. As $T>0$ the probabilities $P_{\nu_{e} \rightarrow \nu_{\mu}}$ and $P_{\nu_{e} \rightarrow \nu_{\tau}}$ increase and oscillate while remaining close. Similar considerations hold for states $\left|\nu_{\mu}(t)\right\rangle$ and $\left|\nu_{\tau}(t)\right\rangle$. Entanglement and flavor transition probabilities for these states exhibit very fast oscillating behaviors, related to the experimentally measured values of the mixing parameters.

Because of $C P T$ invariance, the $C P$ asymmetry $\Delta_{C P}^{\alpha, \beta}$ is equal to the asymmetry under time reversal, defined as

$$
\begin{aligned}
\Delta_{T}^{\alpha, \beta} & =P_{\nu_{\alpha} \rightarrow \nu_{\beta}}(t)-P_{\nu_{\beta} \rightarrow \nu_{\alpha}}(t) \\
& =P_{\nu_{\alpha} \rightarrow \nu_{\beta}}(t)-P_{\nu_{\alpha} \rightarrow \nu_{\beta}}(-t) .
\end{aligned}
$$

In the three-flavor instance, such a quantity is different from zero for a nonvanishing phase $\delta$. It is worth noticing that $\sum_{\beta} \Delta_{C P}^{\alpha \beta}=0$ with $\alpha, \beta=e, \mu, \tau$. Introducing the "imbalances", i.e. the difference between the linear entropies and their time-reversed expressions:

$$
\Delta S_{L \lambda}^{(\alpha, \beta ; \gamma)}=S_{L \lambda}^{(\alpha, \beta ; \gamma)}(t)-S_{L \lambda}^{(\alpha, \beta ; \gamma)}(-t),
$$

we can compute, e. g., $\Delta S_{L e}^{(e, \mu ; \tau)}$, and obtain:

$$
\Delta S_{L e}^{(e, \mu ; \tau)}=4 \Delta_{C P}^{e, \mu}\left(\left|\widetilde{\mathbf{U}}_{e \tau}(t)\right|^{2}+\left|\widetilde{\mathbf{U}}_{\tau e}(t)\right|^{2}-1\right),
$$

where the last factor is $C P$-even. In Fig. [ we show the behavior of the imbalances $\Delta S_{L e}^{(\alpha, \beta ; \gamma)}$ as functions of time, and see how they effectively measure $C P$ violation.

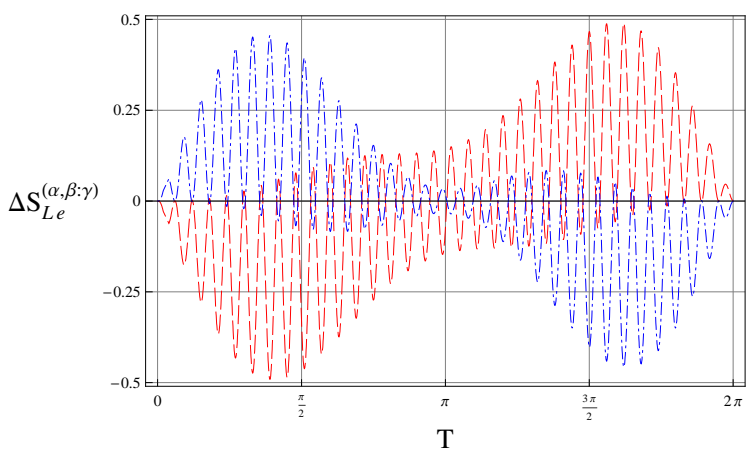

Fig. 4: (Color online) The imbalances $\Delta S_{L e}^{(\alpha, \beta ; \gamma)}$ as functions of the scaled time $T$. Curves correspond to $\Delta S_{L e}^{(e, \mu ; \tau)}$ (longdashed) and $\Delta S_{L e}^{(e, \tau ; \mu)}$ (dot-dashed). The quantity $\Delta S_{L e}^{(\mu, \tau ; e)}$ is vanishing. Parameters $\theta_{i j}$ and $\Delta m_{i j}^{2}$ are fixed at the central experimental values as in Fig. 2 The $C P$-violating phase is set at the value $\delta=\pi / 2$.

In order to demonstrate that the form of single-particle entanglement encoded in the time-evolved flavor states $\left|\underline{\nu}^{(f)}(t)\right\rangle$ is a real physical resource that can be legitimately used, at least in principle, for protocols of quantum information, we discuss an experimental scheme for the transfer of the flavor entanglement of a neutrino beam into that of a single-particle system with spatially separated modes. For simplicity, we will restrict the analysis to two flavors $\alpha=e, \mu$. Consider the elementary charged-current interaction between a neutrino $\nu_{\alpha}$ with flavor $\alpha$ and a nucleon $N$ [13]. The quasi-elastic scattering interaction yields the production of a lepton $\alpha^{-}$and of an outgoing baryon $X$, according to the reaction:

$$
\nu_{\alpha}+N \longrightarrow \alpha^{-}+X
$$

In the simplest instance, the nucleon $N$ is a neutron and the baryon $X$ is a proton $p$; the corresponding scheme is 
Entanglement in neutrino oscillations

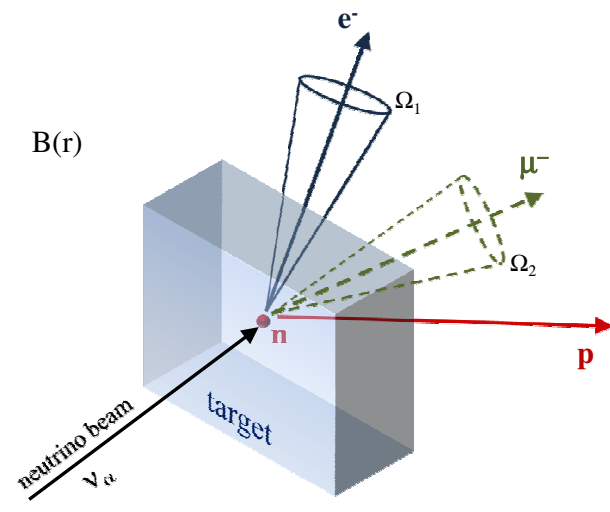

Fig. 5: (Color online) Scheme for the generation of singleparticle entangled lepton states. A neutrino beam is focused on a target where events of the following charged-current interaction may occur: $\nu_{\alpha}+n \longrightarrow \alpha^{-}+p$ with $\alpha=e, \mu$. The beam is assumed to have energy higher than the threshold value necessary for the creation of a muon. A spatially nonuniform magnetic field $\mathbf{B}(\mathbf{r})$ is then applied to limit the momentum of the outgoing lepton within a certain solid angle $\Omega_{i}$, and to ensure the spatial separation between the electron and muon spatial paths. The reaction produces a superposition of electronic and muonic spatially separated states.

illustrated in Fig. 5. Given the initial Bell-like superposition (7), the unitary process associated with the weak interaction (17) produces the superposition

$$
|\alpha(t)\rangle=\Lambda_{e}|1\rangle_{e}|0\rangle_{\mu}+\Lambda_{\mu}|0\rangle_{e}|1\rangle_{\mu},
$$

where $\left|\Lambda_{e}\right|^{2}+\left|\Lambda_{\mu}\right|^{2}=1$, and $|k\rangle_{\alpha}$, with $k=0$, 1 , represents the lepton qubit. The coefficients $\Lambda_{\alpha}$ are proportional to $\widetilde{\mathbf{U}}_{\alpha \beta}(t)$ and to the cross sections associated with the creation of an electron or a muon. Comparing our singlelepton system with the single-photon system, the quantum uncertainty on "which path" of the photon at the output of an unbalanced beam splitter is replaced by the uncertainty on "which flavor" of the produced lepton. The coefficients $\Lambda_{\alpha}$ play the role of the transmissivity and of the reflectivity of the beam splitter. Moreover, by exploiting the mass difference between the two leptons, the desired spatial separation between the flavors can be achieved by applying a nonuniform magnetic field. It is also important to remark that the approach proposed in the present work can be applied even in extended neutrino models including one or more sterile neutrinos. In such cases, from a mathematical point of view the main difference is that one deals with more than three modes (flavors), while, from an operational point of view, the presence of sterile neutrinos (undetectable to date) would introduce a mechanism of loss of quantum information by making the (observed) mixing matrix non unitary. Therefore we can conclude that, at least in principle, the quantum information encoded in the neutrino flavor states can be transferred to a delocalized two-flavor lepton state, and the single-particle mode entanglement acquires an operational characterization that can be exploited for quantum information tasks using systems of elementary particle physics.

$$
* * *
$$

We acknowledge financial support from MIUR, CNRINFM Research and Development Center "Coherentia", INFN, and from ISI Foundation for Scientific Interchange.

\section{REFERENCES}

[1] Nielsen M. A. and Chuang I. L., Quantum Computation and Quantum Information (CUP, Cambridge, UK) 2001.

[2] Horodecki M., Horodecki P. and Horodecki R., Phys. Rev. A , 60 (1999) 1888.

[3] Adesso G. and Illuminati F., Phys. Rev. Lett. , 95 (2005) 150503.

[4] Zanardi P., Phys. Rev. A , 65 (2002) 042101; Shi Y., Phys. Rev. A , 67 (2003) 024301; Zanardi P., Lidar D. A. and Lloyd S., Phys. Rev. Lett. , 92 (2004) 060402;

[5] VAn Enk S. J., Phys. Rev. A, 72 (2005) 064306; ibid., 74 (2006) 026302;

[6] Terra Cunha M. O.,Dunningham J. A. and Vedral V., Proc. Royal Soc. A , 463 (2007) 2277.

[7] Buörk G., Jonsson P. and Sánchez-Soto L. L., Phys. Rev. A, 64 (2001) 042106;

[8] Lee J. W. et al., Phys. Rev. A , 68 (2003) 012324; NhA H. and Kim J., Phys. Rev. A , 75 (2007) 012326;

[9] Lombardi E. et al., Phys. Rev. Lett., 88 (2002) 070402;

[10] Lvovsky A. I. et al., Phys. Rev. Lett., 87 (2001) 050402; Babichev S. A. , Appel J. and Lvovsky A. I., Phys. Rev. Lett., 92 (2004) 193601;

[11] Hessmo B. et al., Phys. Rev. Lett., 92 (2004) 180401;

[12] Dunningham J. and Vedral V. , Phys. Rev. Lett., 99 (2007) 180404;

[13] Cheng T. and Li L. , Gauge Theory of Elementary Particle Physics (Clarendon Press) 1989.

[14] Casas J. A. and Ibarra A., Nucl. Phys. B, 618 (2001) 171 ;

[15] Blasone M. and Vitiello G., Ann. Phys. (N.Y.), 244 (1995) 283; Blasone M., JizBa P. and Vitiello G., Phys. Lett. B, 517 (2001) 471.

[16] Blasone M., Dell'Anno F., De Siena S., Di Mauro M. and Illuminati F. , Phys. Rev. D , 77 (2008) 096002.

[17] Dür W., Vidal G. and Cirac J. I. , Phys. Rev. A, 62 (2000) 062314.

[18] Amico L., Fazio R., Osterloh A. and Vedral V., Rev. Mod. Phys., 80 (2008) 517; Horodecki R., Horodecki P., Horodecki M. and Horodecki K., quant-ph/0702225.

[19] Meyer D. A. and Wallach N. R., J. Math. Phys. , 43 (2002) 4273.

[20] Brennen G. K., Quantum Inf. Comp., 3 (2003) 619.

[21] de Oliveira T. R., Rigolin G. and De Oliveira M. C., Phys. Rev. A, 73 (2006) 010305(R).

[22] Wei T. C. and Goldbart P. M., Phys. Rev. A, 68 (2003) 042307.

[23] Blasone M., Dell'Anno F., De Siena S. and Illuminati F., Phys. Rev. A, 77 (2008) 062304.

[24] Fogli G. L. et al., Phys. Rev. D , 75 (2007) 053001. 